\title{
Comparação do alongamento estático, balístico e contrair-relaxar nos músculos isquiotibiais
}

\author{
Comparison of static, ballistic and contract-relax stretching in hamstring muscle
}

Comparación del estiramiento estático, balístico

y contrae-relaja en los músculos isquiocrurales

\author{
Mary Hellen Morcelli', Júlia Martins Cruz Alves Oliveira², Marcelo Tavella Navega²
}

RESUMOIObjetivou-se, por meio deste estudo, comparar o efeito de três técnicas de alongamento muscular (balístico, estático e contrai-relaxa) no ganho imediato de flexibilidade dos músculos isquiotibiais. Foram avaliados 23 sujeitos, de ambos os gêneros [média (DP) idade 21,17 (1,4)]. Todos os voluntários realizaram o alongamento estático, o balístico e o contrai-relaxa. Cada técnica foi realizada em sessão única com intervalo de sete dias entre as sessões. Para avaliar os efeitos imediatos das diferentes técnicas, realizaram-se, antes e após a série de alongamentos, os testes de flexibilidade Sentar e Alcançar e o Teste do Ângulo Poplíteo. Em comparação à linha de base, houve aumento significativo de flexibilidade para o teste do Ângulo Poplíteo após aplicação do alongamento balístico (6,26\%) e contrai--relaxa (6,5\%), respectivamente $(p<0,05)$. Ao contrair-relaxar as três técnicas em relação ao escore de mudança, encontrou-se diferença considerável no teste do Ângulo Poplíteo para os alongamentos balístico e contrair-relaxar, porém, o Teste Sentar e Alcançar não apresentou relevância. De acordo com esses dados, as técnicas balísticas e contrair-relaxar foram melhores que a técnica de alongamento estático, e ambas as técnicas mostraram-se igualmente efetivas. As técnicas de alongamento balístico e contrair-relaxar melhoraram a flexibilidade dos músculos isquiotibiais.

Descritores I amplitude de movimento articular; exercícios de alongamento muscular biomecânica.

ABSTRACTI This study aimed to compare the effect of three stretching techniques (ballistic, static and contract-relax) on instant gain muscle flexibility on the hamstring muscle. We evaluated 23 subjects of both gender [means (SD) age 21.17 (1.4) years]. All volunteers performed static, ballistic and contractrelax stretching. Each technique was performed in a single session with an interval of seven days between sessions. To assess the immediate effects of the different techniques were performed two tests of flexibility, Sit and Reach Test and the Popliteal Angle Test. In comparison with the baseline there were significant increase in muscle flexibility in the Popliteal Angle Test after application ballistic (6.26\%) and contract-relax technique (6.5\%) respectively $(p<0.05)$. When comparing the three techniques regarding the change score significant difference was found in the the Popliteal Angle Test for ballistic and contract-relax stretching, but Sit and Reach Test showed no significance difference. These data suggest that the ballistic and contract-relax were better than the technique of static stretching and both are equally effective. The ballistic contractrelax stretching techniques improved gain of muscle flexibility on the hamstring muscle.

Keywords I range of motion muscle stretching; exercises biomechanics.

RESUMEN I Se procuró, por medio de este estudio, comparar el efecto de tres técnicas de alongamiento muscular (balístico, estático y contrae-relaja) en el aumento inmediato de flexibilidad de los músculos isquiocrurales. Se evaluaron 23 sujetos, de ambos géneros [media (DE) edad 21,17 (1,4)]. Todos voluntarios realizaron el alongamiento estático, el balístico y el contrae-relaja. Cada técnica fue realizada en sesión única con intervalo de siete días entre las sesiones. Para evaluar los efectos inmediatos de las diferentes técnicas, se realizaron, antes y después de la serie de alongamientos, los tests de flexibilidad Sentar y Alcanzar y el Test del Ángulo Poplíteo. En comparación a la línea de base, hubo aumento significativo de flexibilidad para el test del Ángulo Poplíteo, después de aplicación del alongamiento balístico (6,26\%) y contraerelaja (6,5\%), respectivamente $(p<0,05)$. Al comparar las tres técnicas en relación al escore de cambio, se encontró diferencia considerable en el test del Ángulo Poplíteo para los

Estudo desenvolvido na Faculdade de Filosofia e Ciências da Universidade Estadual Paulista “Júlio de Mesquita Filho" (UNESP) - Marília (SP), Brasil. 'Departamento de Educação Física, UNESP - Rio Claro (SP), Brasil.

²Departamento de Fisioterapia e Terapia Ocupacional, UNESP - Marília (SP), Brasil.

Endereço para correspondência: Mary Hellen Morcelli - Universidade Estadual Paulista, Departamento de Educação Física - Avenida 24-A, 1515 - Bela Vista - CEP: 13506-900 Rio Claro (SP), Brasil -E-mail: mary_morcelli@yahoo.com.br

Apresentação: out. 2012 - Aceito para publicação: jul. 2013 - Fonte de financiamento: nenhuma - Conflito de interesses: nada a declarar - Apresentação em evento científico: XIX Congresso Brasileiro de Fisioterapia-AFB 2011, Florianópolis - Parecer de aprovação no Comitê de Ética nº 0527/2010. 
alongamientos balístico y contrae-relaja, sin embargo el Test Sentar y Alcanzar no presentó relevancia. De acuerdo con esos datos, las técnicas balísticas y contrae-relaja fueron mejores que la técnica de alongamiento estático y, ambas técnicas, se mostraron igualmente efectivas. Las técnicas de alongamiento balístico y contrae-relaja mejoraron la flexibilidad de los músculos isquiocrurales.

Palabras clave I amplitud de movimiento articular; ejercicios de alongamiento muscular biomecánica.

\section{INTRODUÇÃO}

Há muitas consequências causadas pelo encurtamento dos músculos isquiotibiais, entre elas, os desvios posturais ${ }^{1}$, a mudança no ritmo lombo-pélvico, dor na parte inferior das $\operatorname{costas}^{1,2}$, predisposição a lesões ${ }^{3}$ e desenvolvimento de tendinite patelar ${ }^{2}$. Levando em consideração esta realidade, a realização de exercícios de alongamento promove alguns benefícios, como a melhor performance atlética e ganhos funcionais, além da manutenção da saúde e boa forma ${ }^{4,5}$.

As principais técnicas de alongamento são: alongamento estático, balístico e contrair-relaxar ${ }^{6}$. O alongamento estático é um método pelo qual os tecidos moles são alongados até o ponto de resistência ou tolerância do tecido mantido nesta posição ${ }^{7}$. O balístico é caracterizado pelo uso de movimentos vigorosos e rítmicos de um segmento do corpo, pelo alcance do movimento, com o objetivo de alongar o músculo ou o grupo muscular ${ }^{8}$,enquanto a técnica do contrair-relaxar usa uma leve contração isométrica do músculo agonista, que inibe o músculo a ser alongado, e depois realiza o alongamento estático durante o relaxamento deste músculo ${ }^{8,9}$.

Alguns estudos sugerem que as técnicas de alongamento contrair-relaxar e balística são mais eficientes em termos de ganho de flexibilidade dos músculos isquiotibiais em relação à técnica de alongamento estático. Por outro lado, estudos mostram que o alongamento estático pode ser o único capaz de manter um ganho significativo na amplitude dos músculos isquiotibiais por um longo período de tempo ${ }^{4,6}$.

A importância dos estudos relacionados ao encurtamento dos músculos isquiotibiais se deve à possibilidade da existência de lesões causadas por mudanças biomecânicas, que podem levar a distúrbios, como disfunção patelofemoral, dor no púbis, dor nas costas, tendinite patelar e problemas posturais ${ }^{1}$. As lesões dos músculos isquiotibiais somam $29 \%$ de todas as contusões em atletas, e de 12 a $31 \%$ desses atletas sofrem com a reincidência das mesmas. Além disso, considerando que, na prática clínica, é comum utilizar protocolos de alongamento, a literatura deveria apresentar estudos comparativos dos efeitos de tais protocolos, avaliando as vantagens e desvantagens das técnicas de alongamento, incluindo o estático, o balístico e o contrair-relaxar ${ }^{10}$.

Então, os objetivos deste estudo foram: analisar a efetividade das técnicas de alongamento estático, balístico e contrair-relaxar e comparar os efeitos imediatos destas técnicas na flexibilidade dos músculos isquiotibiais.

\section{METODOLOGIA}

\section{Participantes}

Vinte três estudantes universitários, de ambos os sexos, com idades entre 18 e 25 anos, foram avaliados (Tabela 1). Os voluntários foram instruídos a não praticar alongamento e/ou sessões de esforço muscular envolvendo os membros inferiores nos últimos três meses antes do estudo. Indivíduos que reportaram lesões em músculos isquiotibiais nos últimos três meses e aqueles que estavam sob uso constante de analgésicos durante as duas últimas semanas foram excluídos da amostra. Todos os voluntários assinaram um termo de consentimento livre e esclarecido.

\section{Procedimentos}

As três técnicas de alongamento foram realizadas por todos os voluntários. Cada técnica foi realizada em uma sessão única, com um intervalo de sete dias entre as sessões, em ordem aleatória. Ao avaliar a flexibilidade, o Teste de Sentar e Alcançar (TSA) e o Teste do Ângulo Poplíteo (AP) foram utilizados. Ambos os testes, quando utilizaram a mesma amostra do presente estudo, mostraram, em análise intraobservador, um coeficiente de correlação intraclasse (CCI) de 0,91 e 0,79, respectivamente ${ }^{11}$. Testes e técnicas de alongamento foram realizados pelo mesmo avaliador, com experiência prévia. Cada teste foi aplicado antes e depois da conclusão de cada técnica.

O TSA, que avalia a flexibilidade da cadeia muscular posterior, foi realizado com base em estudos prévios ${ }^{12,13}$.

Tabela 1. Características dos participantes

$\begin{array}{lccc}\text { Variávels } & \begin{array}{c}\text { Homens }(n=7) \\ \text { Média } \pm D P\end{array} & \begin{array}{c}\text { Mulheres }(n=16) \\ \text { Média } \pm D P\end{array} & \begin{array}{c}\text { Total }(n=23) \\ \text { Média } \pm D P\end{array} \\ \text { Idade (anos) } & 20,85 \pm 1,57 & 21,31 \pm 1,35 & 21,17 \pm 1,40 \\ \text { Massa corporal }(\mathrm{kg}) & 75,71 \pm 9,06 & 57,09 \pm 6,67 & 62,76 \pm 11,38 \\ \text { Altura }(\mathrm{m}) & 1,75 \pm 0,04 & 1,59 \pm 0,04 & 1,64 \pm 0,08 \\ \text { IMC }\left(\mathrm{kg} / \mathrm{m}^{2}\right) & 24,76 \pm 3,91 & 22,33 \pm 2,32 & 23,07 \pm 3,03\end{array}$

DP: desvio-padrão; IMC: índice de massa corporal 
O indivíduo era instruído a se sentar na maca, com os joelhos estendidos, e flexionar os quadris com o cotovelo estendido. $\mathrm{O}$ participante deveria alcançar a maior distância possível sem dobrar os joelhos.

Para realizar o AP, marcadores de isopor foram $\mathrm{co}^{-}$ locados em proeminências ósseas do grande trocanter, epicôndilo lateral do fêmur e maléolo lateral no membro dominante do voluntário ${ }^{14}$. $\mathrm{O}$ membro contralateral foi estabilizado em extensão total, e o membro dominante foi estabilizado a $90^{\circ}$ da flexão do quadril, com o joelho relaxado. $\mathrm{O}$ goniômetro foi posicionado com o braço imóvel em direção ao grande trocanter do fêmur, e o braço móvel em direção ao maléolo lateral. Solicitou-se que o voluntário estendesse seu joelho até que se sentisse desconfortável. Ambos os testes foram realizados três vezes, e a média das três medidas foi usada para a análise de dados.

A ordem de aplicação de cada técnica de alongamento foi selecionada aleatoriamente (Figura 1).

- Alongamento estático: o quadril do voluntário foi passivamente flexionado pelo examinador até o ponto máximo de flexão, com a articulação do joelho em extensão total. Cinco ciclos de 30 segundos foram realizados com um intervalo de 30 segundos entre eles.

- Alongamento contrair-relaxar: O quadril do voluntário foi passivamente flexionado pelo examinador até o ponto máximo de flexão, com a articulação do joelho em extensão total. Foram realizados cinco ciclos de trinta segundos, cinco segundos de contração isométrica e dez segundos de alongamento com os músculos relaxados. Este processo foi realizado duas vezes para que o ciclo de trinta segundos fosse concluído. Houve um intervalo de trinta segundos entre os ciclos.

- Alongamento balístico: movimentos de flexão e extensão do quadril, com o joelho em extensão total. Os movimentos foram realizados pelo examinador, o mais rápido possível, respeitando o limite de cada voluntário. Foram realizados trinta ciclos de trinta segundos, com trinta segundos de intervalo entre eles.

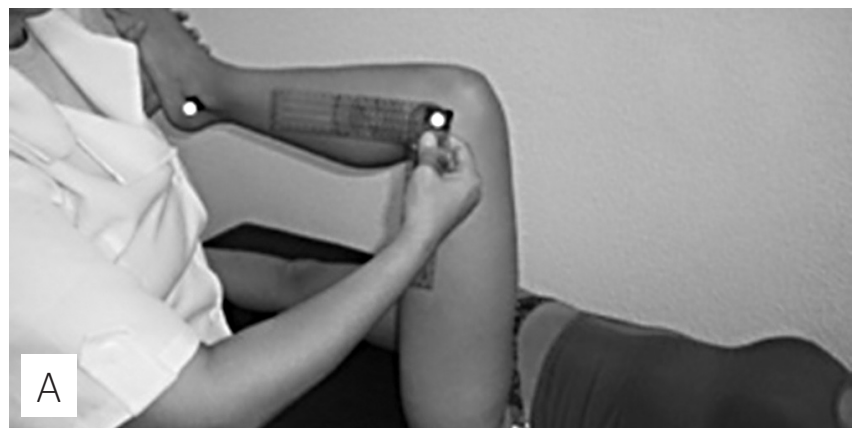

Figura 1. (A) Teste do Ângulo Poplíteo; (B) Teste Sentar e Alcançar

\section{Análise estatística}

A análise dos dados utilizou o software SAS para cálculos de estatísticas analíticas. A normalidade da distribuição dos dados foi testada por meio do teste de Shapiro-Wilk. Para comparar dados antes e depois do alongamento, o teste ANOVA two-way com medidas repetidas (análise de variância) foi usado, seguido do teste post hoc de Tukey. Além disso, os dados foram reorganizados de acordo com o dia. Esses dados foram reorganizados para checar se o ganho de flexibilidade, independentemente da técnica, era duradouro. Para comparar os dados de acordo com o dia, o teste ANOVA two-way com medidas repetidas foi seguido do teste post hoc de Tukey. Os escores de variação entre as três técnicas de alongamento foram realizados pela análise de variância de Friedman (ANOVA), seguida pelo teste de Student Newman-Keus, já que as variâncias não foram homogêneas. $\mathrm{O}$ nível de significância adotado para a análise dos dados foi $5 \%(\mathrm{p}<0,05)$.

\section{RESULTADOS}

O TSA não apresentou aumento significativo da flexibilidade após a aplicação do alongamento estático $(\mathrm{p}=0,59$; $f=0,29)$, balístico $(p=0,63 ; f=0,22)$ e contrair-relaxar $(\mathrm{p}=0,46 ; \mathrm{f}=0,5)($ Tabela 2$)$.

Tabela 2. Teste do Ângulo Poplíteo e Teste Sentar e Alcançar antes e depois da realização das técnicas de alongamento: estático, balístico e contrair-relaxar

\begin{tabular}{lcccc} 
& \multicolumn{2}{c}{ Teste do ângulo popliteo (nível) } & \multicolumn{2}{c}{ Teste Sentar e Alcançar (cm) } \\
\cline { 2 - 5 } & $\begin{array}{c}\text { Antes } \\
\text { Média } \pm \text { DP }\end{array}$ & $\begin{array}{c}\text { Depois } \\
\text { Média } \pm \text { DP }\end{array}$ & $\begin{array}{c}\text { Antes } \\
\text { Média } \pm D P\end{array}$ & $\begin{array}{c}\text { Depois } \\
\text { Média } \pm D P\end{array}$ \\
AE & $135,87 \pm 11,83$ & $141,49 \pm 11,4$ & $21,73 \pm 10,83$ & $23,41 \pm 10,51$ \\
AB & $135,03 \pm 12,06$ & $143,49 \pm 11,68^{*}$ & $22,49 \pm 9,77$ & $23,88 \pm 10,16$ \\
ACR & $134,65 \pm 10,81$ & $143,4 \pm 10,99^{* *}$ & $22,72 \pm 10,42$ & $24,89 \pm 10,32$
\end{tabular}

AE: Alongamento estático; AB: Alongamento balístico; ACR: Alongamento contrair-relaxar; DP: desvio-padrão

* diferença significante antes e depois da aplicação da técnica de alongamento balístico ** diferença significante antes e depois da aplicação da técnica de alongamento contrair-relaxar

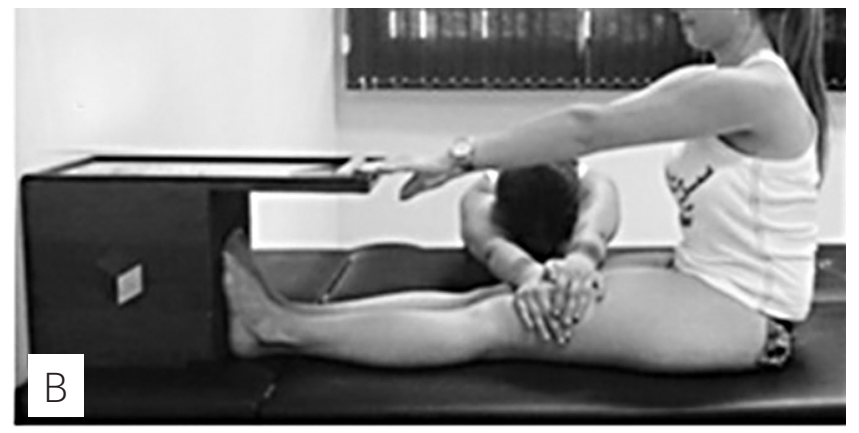


Tabela 3. Escore de variação do Teste Sentar e Alcançar e do Teste do Ângulo Poplíteo para as três técnicas de alongamento aplicadas.

$\begin{array}{lcccc} & \text { Alongamento estático } & \text { Alongamento balístico } & \text { Alongamento contrair-relaxar } & \text { Valor } p \\ \text { Teste Sentar e } & 1,13(0,03-5,63) & 1,17(0-5,33) & 2,1(0-6,03) & 0,66 \\ \text { Alcançar (cm) } & & & 9,01 \\ \text { Teste do Ângulo } & 5,33(0-16) & 8,67(1,67-3,33) & 9(1-17,66) & <0,05^{\star} \\ \text { Poplíteo (níveis) } & & & <0,05^{* *}\end{array}$

* p<0,05 em relação ao alongamento estático e balístico; ** p<0,05 em relação ao alongamento estático e contrair-relaxar; Valores apresentados em mediana (mínimo-máximo)

Tabela 4. Valores iniciais do Teste Sentar e Alcançar e do Teste do Ângulo Poplíteo em três dias, independentemente da técnica de alongamento aplicada

$\begin{array}{lcc} & \begin{array}{c}\text { Teste Sentar e Alcançar } \\ (\mathrm{cm}) \text { Média } \pm \text { DP }\end{array} & \begin{array}{c}\text { Teste do Ângulo Poplíteo } \\ \text { (níveis) Média } \pm \text { DP }\end{array} \\ \text { 10 Dia } & 21,8 \pm 11,0 & 134,7 \pm 11,4 \\ \text { 20 Dia } & 22,1 \pm 9,8 & 135,9 \pm 10,9 \\ \text { 30 Dia } & 22,8 \pm 10,1 & 134,9 \pm 12,3 \\ \text { Valor de P } & 0,94 & 0,93 \\ \text { Valor de f } & 0,61 & 0,71\end{array}$

DP: desvio-padrão

Diferenças significativas no alongamento balístico $(6,26 \%)$ IP $=0,01 ; \mathrm{f}=5,84)$ e contrair-relaxar $(6,5 \%)$ ( $\mathrm{p}=0,009 ; \mathrm{f}=7,41)$ foi observado no AP (Tabela 2$)$.

Não houve diferença significativa entre os valores iniciais comparando as três técnicas, assim como entre os valores finais, tanto no TSA ( $\mathrm{p}=0,94, \mathrm{f}=0,58 ; \mathrm{p}=0,88, \mathrm{f}=0,2$, respectivamente) e no $\mathrm{AP}(\mathrm{p}=0,93, \mathrm{f}=0,67 ; \mathrm{p}=0,79, \mathrm{f}=0,22$ respectivamente) (Tabela 2). Em relação à diferença no escore de variação de cada técnica de alongamento, um nível significativo foi observado no AP ( $\mathrm{p}=0,01)$ quando comparamos as técnicas de alongamento balístico e estático $(\mathrm{p}<0,05)$, assim como o estático e o contrair-relaxar ( $\mathrm{p}<0,05)$; porém, não houve diferenças quando as técnicas contrair-relaxar e balística foram comparadas $(p=0,09)$. Também não houve diferença entre os valores final e inicial de cada técnica de alongamento aplicada ao TSA $(\mathrm{p}=0,66)$ (Tabela 3$)$.

Os valores iniciais do TSA e do AP em cada dia (D1, D2, D3), independentemente da técnica de alongamento, estão demonstrados na Tabela 4. Não houve diferença estatística significativa para estes valores.

\section{DISCUSSÃO}

O objetivo deste estudo foi comparar o ganho imediato de flexibilidade dos músculos isquiotibiais após a aplicação de técnicas de alongamento estático, balístico e contrair-relaxar. Os resultados do AP sugerem que o alongamento balístico e o contrair-relaxar são capazes de aumentar a imediata flexibilidade dos músculos isquiotibiais. Esses dados corroboram os achados de outros estudos sobre a implementação de protocolos de alongamento para aumentar a flexibilidade de tais músculos ${ }^{15,16}$.

Há muitas técnicas que avaliam as mudanças na flexibilidade muscular com base nas articulações e movimentos a serem analisados. Por isso, testes funcionais usados para analisar a flexibilidade de músculos isquiotibiais podem incluir movimentos de extensão de joelho e flexão do tronco na articulação do quadril ${ }^{12}$. O AP mede a flexibilidade dos músculos isquiotibiais ${ }^{17}$ e demonstra ser confiável quando realizado em sujeitos saudáveis ${ }^{18}$.

O TSA também prova ser confiável para avaliar a flexibilidade dos músculos isquiotibiais ${ }^{19,20}$, mas também mede a flexibilidade do segmento corporal inferior $^{19}$. AP pode ser mais sensível às mudanças na flexibilidade dos músculos isquiotibiais após a aplicação das três técnicas de alongamento.

Este ganho imediato de flexibilidade pode ser explicado pelas propriedades viscoelásticas do músculo. Esta característica é uma resposta da unidade músculo-tendão ao alongamento com comprimento fixo, que leva à diminuição da força com o tempo. Este comprimento reduzido da unidade músculo-tendão é conhecido como relaxamento de força, e permite a distorção da unidade músculo-tendão mais facilmente na repetição seguinte, promovendo mais flexibilidade ${ }^{16}$. Outra explicação é baseada na maior tolerância da unidade músculo-tendão ao alongamento, embora este mecanismo ainda seja desconhecido ${ }^{21}$.

Além disso, o contrair-relaxar também pode ser explicado por fatores neurofisiológicos, baseados em descobertas envolvendo a inibição recíproca e a indução subsequente. A inibição recíproca descreve o fenômeno pelo qual, enquanto um grupo muscular é ativado, seu antagonista é inibido, e isso facilita o alongamento desta unidade músculo-tendão ${ }^{22}$. Em relação aos ganhos de flexibilidade observados após a aplicação do alongamento balístico, a literatura demonstra que os movimentos realizados neste tipo de alongamento podem aquecer a musculatura corporal. $\mathrm{O}$ aumento da temperatura do corpo durante o esforço pode levar à 
diminuição da viscosidade nos tecidos e retificar as ondulações de colágeno ${ }^{23}$.

Os resultados do escore de variação do AP sugerem que o alongamento balístico e o contrair-relaxar são igualmente eficazes e melhores que o alongamento estático, embora não haja diferença significativa entre as técnicas balística e contrair-relaxar em relação ao ganho imediato de flexibilidade nesses músculos.

Alguns estudos mostraram efeitos similares ao deste estudo em relação à flexibilidade de músculos isquiotibiais, e encontraram poucas melhorias significativas na flexibilidade após a implementação das técnicas balística e contrair-relaxar quanto comparadas ao alongamento estático ${ }^{24,25}$. Porém, outros estudos não encontraram diferenças significativas entre o alongamento estático e o contrair-relaxar, ou entre as técnicas estática e balística, em termos de ganho de flexibilidade nos músculos isquiotibiais ${ }^{15,26}$.

A diferença entre os resultados observados neste estudo e aqueles encontrados na literatura se deve à realização de protocolos de alongamento que usam o início da sensação de dor como referência, enquanto neste estudo utilizou-se a tolerância máxima ao alongamento sem dor ${ }^{21}$.

Além disso, essas diferenças podem ter ocorrido devido aos diversos protocolos usados nos estudos, variações nas posições para realizar as técnicas de alongamento, escolha do membro inferior para utilização e também a metodologia de avaliação. Por isso, novas pesquisas incluindo mais participantes, de sexos e idades diferentes, são importantes.

Não houve diferença significativa nos valores iniciais do TSA e AP para cada dia de coleta, independentemente da técnica de alongamento utilizada. Isso demonstra que a realização de qualquer uma dessas técnicas em cinco séries de 30 segundos, somente uma vez por semana, não é suficiente para ganhar flexibilidade duradoura.

Neste estudo, não foi observada interferência do acúmulo de exercícios de alongamento na avaliação inicial, que aconteceu no segundo e no terceiro dias, já que não houve diferença estatisticamente significativa nos valores iniciais de cada dia para ambos os testes, independentemente da técnica de alongamento aplicada.

Em termos de limitações do estudo, o número de participantes foi pequeno, então a extrapolação dos dados para populações e grupos musculares diferentes deve ser cuidadosa. Mais ensaios randomizados e controlados devem ser realizados para estabelecer os benefícios específicos do alongamento estático, balístico e contrair-relaxar.

\section{CONCLUSÃO}

Os resultados deste estudo preliminar sugerem que as técnicas balística e contrair-relaxar podem melhorar o ganho imediato de flexibilidade dos músculos isquiotibiais. O alongamento balístico e o contrair-relaxar são igualmente eficazes e melhores que o alongamento estático, que não teve efeito sobre o ganho de flexibilidade.

\section{REFERÊNCIAS}

1. Kawano MM, Ambar G, Oliveira BI, Boer MC, Cardoso AP, Cardoso JR. Influence of the gastrocnemius muscle on the sit-and-reach test assessed by angular kinematic analysis. Rev Bras Fisioter. 2010;14(1):10-5.

2. Ylinen J, Kankainen $\mathrm{T}$, Kautiainen $\mathrm{H}$, Rezasoltani A, Kuukkanen $\mathrm{T}$, Häkkinen A. Effect of stretching on hamstring muscle compliance. J Rehabil Med. 2009;41(1):80-4.

3. Ben M, Harvey LA. Regular stretch does not increase muscle extensibility: a randomized controlled trial. Scand J Med Sci Sports. 2010;20(1):136-44

4. Fasen JM, O'Connor AM, Schwartz SL, Watson JO, Plastaras CT, Garvan CW, et al. A randomized controlled trial of hamstring stretching: comparison of four techniques. J Strength Cond Res. 2009;23(2):660-7.

5. Aquino CF, Fonseca ST, Gonçalves GG, Silva PL, Ocarino JM, Mancini MC. Stretching versus strength training in lengthened position in subjects with tight hamstring muscles: a randomized controlled trial. Man Ther. 2010;15(1):26-31.

6. Youdas JW, Haeflinger KM, Kreun MK, Holloway AM, Kramer $\mathrm{CM}$, Hollman JH. The efficacy of two modified proprioceptive neuromuscular facilitation stretching techniques in subjects with reduced hamstring muscle length. Physiother Theory Pract.2010;26(4):240-50.

7. Andrews JR, Harrelson GL, Wilk KE. Reabilitação física das lesões desportiva. Rio de Janeiro: Guanabara Koogan; 2000.p.135-162.

8. Bandy WD, Irion JM, Briggler M. The effect of TSAtic stretch and dynamic range of motion training on the flexibility of the hamstring muscles. J Orthop Sports Phys Ther. 1998;27(4):295-300.

9. Gama ZAS, Medeiros CAS, Dantas AVR, Souza TO. Influência da freqüência de alongamento utilizando facilitação neuromuscular proprioceptiva na flexibilidade dos músculos isquiotibiais. Rev Bras Med Esporte. 2007;13(1):33-8.

10. Shadmehr A, Hadian MR, Naiemi SS, Jalaie S. Hamstring flexibility in young women following passive stretch and muscle energy technique. J Back Musculoskelet Rehabil. 2009;22(3):143-8.

11. Atamaz F, Ozcaldiran B, Ozdedeli S, Capaci K, Durmaz B. Interobserver and intraobserver reliability in lower-limb flexibility measurements. J Sports Med Phys Fitness. 2011; 51(4):689-94.

12. Polachini LO, Fusazaki L, Tamaso M, Tellini GG, Masiero D. Estudo comparativo entre três métodos de avaliação do encurtamento de musculatura posterior de coxa. Rev Bras Fisioter. 2005;9(2):187-93. 
13. Baltaci G, Un N, Tunay V, Besler A, Gerçeker S. Comparison of three different sit and reach tests for measurement of hamstring flexibility in female university students. $\mathrm{Br} J$ Sports Med. 2003;37(1):59-61.

14. Branco VR, Negrao Filho RF, Padovani CR, Barbieri CH, Mazzer N. Relação entre a tensão aplicada e a sensação de desconforto nos músculos isquiotibiais durante o alongamento. Rev Bras Fisioter. 2006:10(4):465-72.

15. Shadmehr A, Hadian MR, Naiemi SS, Jalaie S. Hamstring flexibility in young women following passive stretch and muscle energy technique. J Back Musculoskelet Rehabil. 2009;22(3):143-8.

16 O'Hora J, Cartwright A, Wade CD, Hough AD, Shum GL. Efficacy of TSAtic stretching and proprioceptive neuromuscular facilitation stretch on hamstrings length after a single session. J Strength Cond Res. 2011; 25(6):1586-91.

17. Malheiros DS, Cunha FM, Lima CLFA. Análise da medida do ângulo poplíteo em crianças de sete a treze anos de idade. Rev Bras Ortop. 1995;30(9):693-8.

18. Ten Berge SR, Halbertsma JP, Maathuis PG, Verheij NP, Dijkstra PU, Maathuis KG. Reliability of popliteal angle measurement: a study in cerebral palsy patients and healthy controls. J Pediatr Orthop. 2007;27(6):648-52.
19. Hui SC, Yuen PY, Morrow JR Jr, Jackson AW. Comparison of the criterion-related validity of sit-and-reach tests with and without limb length adjustment in Asian adults. Res Q Exerc Sport. 1999;70(4):401-6.

20 Minkler S, Patterson P. The validity of the modified sit-and-reach test in college-age students. Res Q Exerc Sport.1994;65(2):189-92.

21. Chagas MH, Bhering EL, Bergamini JC, Menzel HJ. Comparação de duas diferentes intensidades de alongamento na amplitude de movimento. Rev Bras Med Esporte. 2008;14(2):99-103.

22 Mitchell UH, Myrer JW, Hopkins JT, Hunter I, Feland JB, Hilton SC. Neurophysiological reflex mechanisms' lack of contribution to the success of PNF stretches. J Sport Rehabil. 2009;18(3):343-57.

23. Gajdosik RL. Passive extensibility of skeletal muscle: review of the literature with clinical implications. Clinical Biomechanics. 2001; 16:87-101.

24. Sousa GGQ, Souza JCC, Trindade-Filho EM, Carvalho ACA. Estudo comparativo da eficácia do alongamento estático em 15,30 e 60 segundos em adultos jovens. Neurobiologia. 2010;73(3):121-30.

25. Azevedo DC, Melo RM, Alves Corrêa RV, Chalmers G. Uninvolved versus target muscle contraction during contract: relax proprioceptive neuromuscular facilitation stretching. Phys Ther Sport. 2011;12(3):117-21.

26. Maddigan ME, Peach AA, Behm DG. A comparison of assisted and unassisted proprioceptive neuromuscular facilitation techniques and TSAtic stretching. J Strength Cond Res. 2012; 26(5):1238-44. 